\title{
Magnetic Fields in the Early Universe
}

\author{
Hector Rubinstein* \\ Stockholm Univ. \\ E-mail: 'rubéphysto.sé
}

Abstract: We discuss some old and new results on mangetic fields

\section{Generalities}

Magnetic fields are everywhere.

Magnetic fields are stable, and hence they could exists since inflation times, or even earlier. Stability is only approximate, a magnetic field will decay if stronger than $B=10^{2} 4$ Gauss. This is so because the Lorentz force cannot perform any work on charged spin half particles so that real particle-antiparticles free pairs cannot be produced. Elementary $\mathrm{W}$ bosons are coupled in a momemtum independent way to the field and can provoke its decay. This is the energy corresponding to creation of a $\mathrm{W}$ pair $10^{24}$ Gauss. We look into this now in some detail. Notice that the electric field is unstable at a comparably very low energy determined by electron positron tunneling. This is the celebrated Schwinger tunneling.

\section{Magnetic fields and the electroweak vacuum}

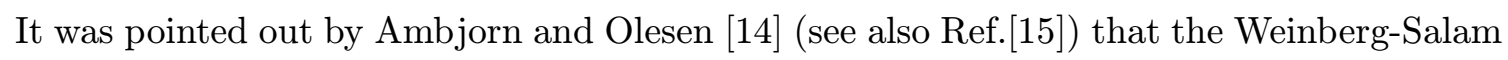
model of electroweak interactions shows an instability at $B \simeq 10^{24}$ Gauss. The nature of such instability can be understood by looking at the expression of the energy of a particle with electric charge $e$, and spin $\mathbf{s}$, moving in homogeneous magnetic field $\mathbf{B}$ directed along the $z$-axis. Above a critical field $B_{c}=m^{2} / e$ particle energy is discretized into Landau levels

$$
E_{n}^{2}=k_{z}^{2}+(2 n+1) e|\mathbf{B}|-2 e \mathbf{B} \cdot \mathbf{s}+m^{2} .
$$

We observe that energy of scalar $(s=0)$ and spinor $\left(s_{z}= \pm 1 / 2\right)$ is always positive, and indeed no instability arise in QED (it is possible to verify that quantum one-loop corrections do not spoil this conclusion). In the case of vector particles $\left(s_{z}=0, \pm 1\right)$,

${ }^{*}$ Speaker. 
however, the lowest energy level $\left(n=0, k_{z}=0, s_{z}=+1\right)$ becomes imaginary for $B>B_{c}$, which could be the signal of vacuum instability. The persistence of imaginary values of

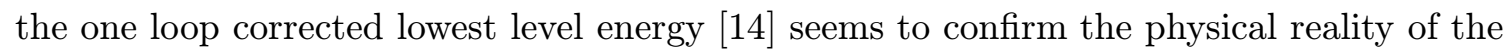
instability.

As it is well known the Weinberg-Salam model contains some charged vector fields, namely the $W^{ \pm}$gauge bosons. The coupling of the $W_{\mu}$ field to an external electromagnetic field $A_{\mu}^{e x t}$ is given by

$$
\mathcal{L}_{i n t}=-\frac{1}{4}\left|F_{\mu \nu}^{e x t}\right|^{2}--\frac{1}{2}\left|D_{\mu} W_{\nu}-D_{\nu} W_{\mu}\right|^{2}-m_{W}^{2} W_{\mu}^{\dagger} W^{\mu}-i e F_{\mu \nu}^{e x t} W^{\mu} W^{\nu}
$$

with

$$
D_{\mu}=\partial_{\mu}-i e A_{\mu}^{e x t} .
$$

The important term in the previous expression is the "anomalous" magnetic moment term $i e F_{\mu \nu}^{e x t} W^{\mu} W^{\nu}$, which arises because the non-Abelian nature of the $S U(2)$ component of the Weinberg-Salam model gauge group structure. Due to this term the mass eigenvalues of the $W$ Lagrangian becomes

$$
m^{2}=m_{W}^{2} \pm e B
$$

As expected from the considerations in the above, a tachyonic mode appears for $B>B_{c}$. The corresponding eigenvector for zero kinetic energy is determined by solving the equation of motion

$$
D_{i} W_{j}-D_{j} W_{i}=0 \quad i, j=x, y,
$$

where $W_{1,2}=W_{x} \pm i W_{y}$. Ambjorn and Olesen argued that a suitable solution of this equation is

$$
|W(x, y)|=e^{-\frac{1}{4} m_{W}\left(x^{2}+y^{2}\right)},
$$

corresponding to a vortex configuration where $W$-fields wind around the $z$-axis. This configuration corresponds to the Nielsen-Olesen vortex solution. A similar phenomenon should also take place for $Z$ bosons. Given the linearity of the equations of motion it is natural to assume that a superpositions of vortices is formed above the critical field. This effect resemble the behaviour of a type-II superconductor in the presence of a critical field magnetic field. In that case $U(1)$ symmetry is locally broken by the formations of a lattice of Abrikosov vortices in the Cooper-pairs condensate through which the field can flow. In the electroweak case this situation is reversed, with the formation of a $W$ condensate along the vortices. Concerning the back-reaction of the $W$ condensate on the magnetic field, an interesting effect arises. By writing the electric current induced by the $W$ fields

$$
j_{\mu}(W)=2 i e\left(W^{\dagger} D_{\mu} W-W D_{\mu} W^{\dagger}\right),
$$

Ambjorn and Olesen noticed that its sign is opposite to the current induced by the Cooper pairs in a type-II superconductor, which is responsible for the Meissner screening effect. Therefore, they concluded that the $W$-condensate induce anti-screening of the external magnetic field. 
Although the Higgs field $\Phi$ does not couple directly to the electromagnetic field (this is different from the case of a superconductor where the Cooper-pairs condensate couples directly to $A_{\mu}^{e x t}$ ), it does through the action of the $W$ condensate. This can be seen by considering the Higgs, $W$ potential in the presence of the magnetic fields:

$$
V(\phi, W)=2\left(e B-m_{W}^{2}\right)|W|^{2}+g^{2} \phi^{2}|W|^{2}-2 \lambda \phi_{0}^{2} \phi^{2}+2 g^{2}|W|^{4}+\lambda\left(\phi_{+}^{4} \phi_{0}^{4}\right) .
$$

In the above $\phi_{0}$ and $\phi_{+}$are respectively the Higgs field vev and charged component, $g$ is the $\mathrm{SU}(2)$ coupling constant, and $\lambda$ is the Higgs the self-interaction coupling constant. We see that the $W$-condensate influences the the Higgs field at classical level due to the $\phi^{2}|W|^{2}$ term. It is straightforward to verify that if $e B<m_{W}^{2}=\frac{1}{2} g^{2} \phi_{0}^{2}$ the minimum of $V(\phi, W)$ sits in the standard field value $\phi=\phi_{0}$ with no $W$ condensate. Otherwise a $W$ condensate is energetically favoured with the minimum of the potential sitting in

$$
\phi_{\min }^{2}=\phi_{0}^{2} \frac{m_{H}^{2}-e B}{m_{H}^{2}-m_{W}^{2}}
$$

where

$$
m_{H}^{2} \equiv 4 \lambda \phi_{0}^{2}, \quad m_{W}^{2} \equiv \frac{1}{2} g^{2} \phi_{0}^{2} .
$$

We see that the Higgs expectation value will vanish as the average strength approaches zero, provided the Higgs mass is larger than the $W$ mass. This seems to suggest that a $W$-condensate should exist for

$$
m_{W}^{2}<e B<m_{H}^{2},
$$

and that the $S U(2) \times U_{Y}(1)$ symmetry is restored above $H_{c}^{(2)} \equiv m_{H}^{2} / e$. Thus, anti-screening should produce restoration of the electroweak symmetry in the core of $W$ vortices. If $m_{H}<m_{W}$ the electroweak vacuum is expected to behave like a type I superconductor with the formation of homogeneous $W$-condensate above the critical magnetic field. The previous qualitative conclusion have been confirmed by analytical and numerical compu-

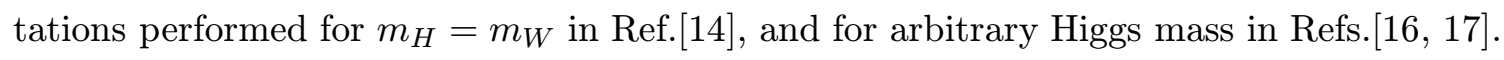

\section{The recent history of cosmic magnetic fields}

The main observational tracers of galactic and extra-galactic magnetic fields are Refs. the Zeeman splitting of spectral lines; the intensity and the polarization of synchrotron emission from free relativistic electrons; the Faraday rotation measurements (RMs) of polarized electromagnetic radiation passing through a ionized medium.

If the magnetic field to be measured is far away one relies on Faraday rotation.

$$
R M\left(z_{s}\right) \equiv \frac{\Delta(\kappa)}{\Delta\left(\lambda^{2}\right)}=8.1 \times 10^{5} \int_{0}^{z_{s}} n_{e} B_{\|}(z)(1+z)^{-2} d l(z) \quad \frac{\mathrm{rad}}{\mathrm{m}^{2}}
$$

where $B_{\|}$is the field strength along the line of sight and

$$
d l(z)=10^{-6} H_{0}^{-1}(1+z)(1+\Omega z)^{-\frac{1}{2}} d z \mathrm{Mpc} .
$$

$H_{0}$ is the Hubble constant. 
We now briefly summarize the observational situation.

Magnetic fields in galaxies. The interstellar field in the Milky Way has been determined using several methods which allowed to obtain valuable information about its amplitude and spatial structure. The average field strength is $3-4 \mu \mathrm{G}$. Such a strength corresponds to an approximate energy equipartition between the magnetic field, the cosmic rays confined in the Galaxy, and the small-scale turbulent motion [i]

$$
\rho_{m}=\frac{B^{2}}{8 \pi} \approx \rho_{t} \approx \rho_{C R} .
$$

Remarkably, the magnetic energy density almost coincides with energy density of the cosmic microwave background radiation (CMBR). The field keeps its orientation on scales of the order of few kiloparsecs (kpc), comparable with the galactic size, and two reversals have been observed between the galactic arms, suggesting that the Galaxy field morphology may be symmetrical. Magnetic fields of similar intensity have been observed in a number of other spiral galaxies. Although equipartition fields were observed in some galaxies, e.g. M33, in some others, like the Magellanic Clouds and M82, the field seems to be stronger than the equipartition threshold. Concerning the spatial structure of the galactic fields, the observational situation is, again, quite confused with some galaxies presenting an axially symmetrical geometry, some other a symmetrical one, and others with no recognizable field structure [inin]

Magnetic fields in galaxy clusters. Observations on a large number of Abel clusters [3i], some of which have a measured X-ray emission, give valuable information on fields in clusters of galaxies. The magnetic field strength in the inter cluster medium (ICM) is well described by the phenomenological equation

$$
B_{I C M} \sim 2 \mu \mathrm{G}\left(\frac{L}{10 k p c}\right)^{-\frac{1}{2}}\left(h_{50}\right)^{-1}
$$

where $L$ is the reversal field length and $h_{50}$ is the reduced Hubble constant. Typical values of $L$ are $10-100 \mathrm{kpc}$ which correspond to field amplitudes of $1-10 \mu \mathrm{G}$. The concrete case of the Coma cluster [4]-4] can be fitted with a core magnetic field $B \sim 8.3 h_{100}^{\frac{1}{2}}$ G tangled at scales of about $1 \mathrm{kpc}$. This is somewhat to be regarded as evidence of primordial fields since comparable values in clusters would imply so. A particular example of clusters with a strong field is the Hydra A cluster for which the RMs imply a $6 \mu \mathrm{G}$ field coherent over

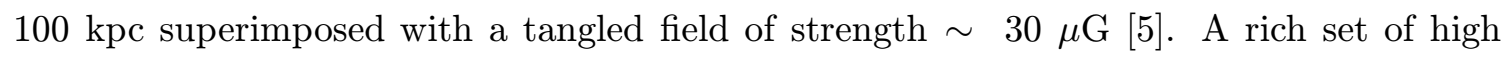
resolution images of radio sources embedded in galaxy clusters shows evidence of strong fields in the cluster central regions [6]. with peak values as large as $\sim 70 \mu \mathrm{G}$. It is noticeable that for such large fields the magnetic pressure exceeds the gas pressure derived from X-ray data suggesting that magnetic fields may play a significant role in the cluster dynamics.It is interesting, as it has been shown by Loeb and Mao [i] that a discrepancy exists between the estimate of the mass of the Abell cluster 2218 derived from gravitational lensing and that inferred from X-ray observations 
which can be well explained by the pressure support produced by a magnetic field with strength $\sim 50 \mu \mathrm{G}$.

\section{Magnetic fields in high redshift objects.}

RMs of the radio emission of the quasar $3 \mathrm{C} 191$, at $z=1.945$ ispresumably due a magnetized shell of gas at the same redshift, are consistent with a field strength in the range $0.4-4 \mu \mathrm{G}$. The field was found to maintain its prevailing direction over at least $\sim 15 \mathrm{kpc}$, which is comparable with a typical galaxy size. The field of a relatively young spiral galaxy at $z=0.395$ was determined by RMs of the radio emission of the quasar PKS 1229-021 lying behind the galaxy at $z=1.038$. The amplitude was firmly estimated to be in the range $1-4 \mu \mathrm{G}$. Even more interesting was the observation of field reversals with distance roughly equal to the spiral arm separation, in a way quite similar to that observed in the Milky Way.

These high fields at early time may be a problem for the dynamo mechanism.

Intergalactic magnetic fields. The radio emission of distant quasars is also used to constrain the intensity of fields in the IGM which we may suppose to pervade the entire Universe. As we discussed, to translate RMs into an estimation of the field strength is quite difficult for rarefied media in which ionized gas density and field coherence length are poorly known. Nevertheless, some interesting limits can be derived on the basis of well known estimates of the Universe ionization fraction and adopting some reasonable values of the magnetic coherence length. For example, assuming a cosmologically aligned magnetic field, as well as $\Omega=1, \Lambda=0$, and $h=0.75$, the RMs of distant quasar imply $B_{I G M}=10^{-11} \mathrm{G}$ [i] As we have seen in the above, in galaxy clusters the largest reversal scale is at most $1 \mathrm{Mpc}$. Adopting this scale as the typical cosmic field coherence length and applying the $\operatorname{RM}\left(z_{s}\right)$ up to $z_{s} \sim 2.5$, Kronberg found the less stringent limit $B_{I G M}=10^{-9} \mathrm{G}$ for the strength at present time.

In conclusion, although the data available today do not allow to answer yet to the question raised in this section, it seems that recent observations and improved theoretical work are putting in question the old wisdom in favour of a dynamo origin of galactic magnetic fields. Especially the recent observations of fieldsz in galaxy clusters suggest that the origin of these fields may indeed be primordial.

\section{The evolution of primordial magnetic fields}

A crucial issue for the investigation of a possible primordial origin of present time galactic and intergalactic fields is that concerning the time evolution of the magnetic fields in the cosmic medium. Three conditions are needed for the persistence of large static fields:

a) intrinsic stability of the field;

b) the absence of free charges which could screen the field; 
c) to have a small diffusion time of the field with respect to the age of the Universe.

Condition a) does not hold for strong electric fields. It is a firm prediction of QED that an

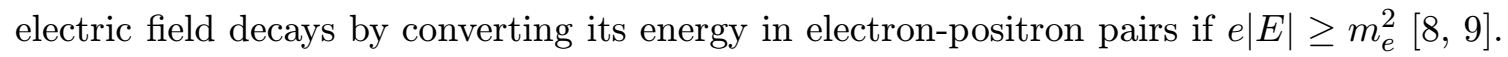
This, however, is a purely electric phenomenon. Only very strong fields may produce nontrivial instabilities in the QCD (if $B>10^{17} \mathrm{G}$ ) and the electroweak vacuum (if $B>$ $10^{23} \mathrm{G}$ ) which may give rise to a partial screening of the field. These effects may have some relevance for processes which occurred at very early times and, perhaps, for the physics of very peculiar collapsed objects like magnetars [i] evolution of cosmic fields after BBN time.

Condition b) is probably trivially fulfilled due to the apparent absence of magnetic monopoles in nature. It is interesting to observe that even a small abundance of magnetic monopoles at present time would have dramatic consequences for the survival of galactic and intergalactic magnetic fields which would lose energy by accelerating the monopoles. This argument was first used by Parker [i] monopole flux, which is $F_{M}=10^{-15} \mathrm{~cm}^{-2} s^{-1} \mathrm{sr}^{-1}$. Also the condition c) does not represent a too serious problem for the survival of primordial magnetic fields. In agreement with the

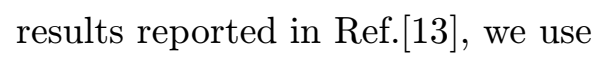

$$
n_{e}(z) \simeq 3 \times 10^{-10} \mathrm{~cm}^{-3} \Omega_{0} h(1+z)^{3},
$$

where $\Omega_{0}$ is the present time density parameter and $h$ is Hubble parameter. Electron resistivity is dominated by Thomson scattering off cosmic background photons. Therefore $\tau \simeq 1 / n_{\gamma} \sigma_{T}$, where $\sigma_{T}=\frac{e^{4}}{6 \pi m_{e}^{2}}$ is the Thomson cross section, and $n_{\gamma}=4.2 \times 10^{2}(1+z)^{3}$. Substituting these expressions we get

$$
\sigma=\frac{n e^{2}}{m_{e} n_{\gamma} \sigma_{T}} \simeq 10^{11} \Omega_{0} h s^{-1} .
$$

It is noticeable that after recombination time Universe conductivity is a constant. Finally, the cosmic diffusion length, i.e. the minimal size of a magnetic configuration which can survive diffusion during the Universe time-life $t_{0}$, is found by substituting $t_{0}=2 \times$ $\left(\Omega_{0} h^{2}\right)^{-1 / 2} \mathrm{~s}^{-1}$ which, adopting $\Omega_{0}=1$ and $h=0.6$, gives

$$
L_{\text {diff }} \simeq 2 \times 10^{13} \mathrm{~cm} \simeq 1 \text { A.U. }
$$

It follows from this result that magnetic diffusion is negligible on galactic and cosmological scales.

\section{Magnetic fields and the photon mass}

We live in a non trivial thermal and perhaps magnetic vacuum. The question is then, do these effects give a mass to the photon? The first point much overlooked is the thermodynamics of photons. Seems that, if there is a mass, Stafan law changes the prefactor due to the number of polarizations, from 2 to 3 irrespective of the mass. Very much like 
in the Higgs effect, longitudinal polarizations are different and it can be shown that the longitudinal waves decouple as

$$
A=\left(\frac{m_{p h}}{E}\right)^{2}
$$

Therefore these components for a mass as the known upper limits of $10^{-17}$ ev would take a part of the lifetime of the Universe to equilibrate in a container!

Nevertheless, the finite temperature of the Universe, requires that necessarily the photon has a thermal mass. It was suggested that it may be large by naively saying that

$$
m_{p} h^{2}=m_{p h}(T=0)^{2}+\theta T^{2}
$$

where $\theta$ is a number proportional to the coupling with the bath. This would give a large mass incompatible with experiment.

Abbott and Gavela [1] via an electron positron loop giving a suppression of order:

$$
e^{-\frac{m_{e}}{k T}}
$$

which is a phenomenally small number.

The next issue, and central to our talk is the following: Can the fact that we live in a magnetic field environment both on earth, galaxy and even perhaps the Universe affect the photon mass?

Adelberger et al $[1 \overline{1} \overline{9}]$ pointed out that the way one introdudes the mass of the photon is model dependent. If one does it by a Higgs mechanism one may have a situation in which the Universe becomes a Type II superconductor. Whether the Higgs cancels the third polarization or not depends on the strength of the field and the mass of he Higgs.

These speculations are interesting but at the present time they just point to the fact that the upper limits of galactic experiments of the photon mass must be interpreted with some care.

The big issue of the origin, extension and strength of cosmic magnetic fields is largely untouched in this review from a theoretical point of view.

It simply reflects the fact that after about thirty years and many papers we still lack a reliable mechanism to generate these fields. Finding some evidence at CMBR times or nucleosynthesis time is possible but difficult. Again, we did not discuss these issues in this talk. Recent reviews by Grasso and Rubinstein and Dolgov discuss some of these issues.

\section{References}

[1] P.P. Kronberg, Rep.Prog.Phys, 57 (1994) 325.

[2] E.G. Zweibel and C. Heiles,Nature3851997131.

[3] K.T. Kim, P.P. Kronberg and P.C. Tribble, Astrophysical Journal

[4] L. Feretti et al., Astronomy and Aastrophysics

[5] G.B. Taylor and R.A. Perley, Astrophysical Journal 
[6] J. Eilek, [astro-ph/9906485].

[7] A. Loeb and S. Mao, Astrophysical Journal 4351994L109.

[8] J. Schwinger, Particles, sources and fields, Vol. 3, Addison-Wesley, 1988.

[9] C. Itzykson and J.B.Zuber, Quantum Field Theory, Mc Graw-Hill Book Co., 1980.

[10] R.F. O'Connell, Phys. Rev. Lett. 211968397.

[11] M.G. Baring and A.K. Harding, [astro-ph/9910127];

[12] E.N. Parker, Astrophyisical Journal, 601970383; ibid. 163 (1971) 225; ibid. 166 (1971) 295.

[13] E.W. Kolb and M.S. Turner, The Early Universe, Addison Wesley (1989).

[14] J. Ambjorn and P. Olesen Nuclear Physics B 151989606; Int.Jour.Mod.Phys.A5(1990), 4525.

[15] V.V. Skalozub, Sov.J.Nucl.Phys. 16 (1985) 445.

[16] V.V. Skalozub, Sov.J.Nucl.Phys. 45 (1987) 1058.

[17] S. MacDowell and O. Törnkvist, Physical Review D, 519923833.

[18] L. Abbott and M Gavela, Nature 20,229, 1980

[19] Eric Adelberger, Gia Dvali, Andrei Gruzinov, hep- hp 0306245 